\title{
Color Separation Gratings for Diverting the Unconverted Light Away from the NIF Target
}

\author{
S. N. Dixit, M. C. Rushford, I. M. Thomas, \\ S. M. Herman, J. A. Britten, B. W. Shore, \\ and M. D. Perry
}

This paper was prepared for submittal to 2nd Annual International Conference on Solid-State Lasers for Applications to Inertial Confinement Fusion Paris, France

October 22-25, 1996

March 11, 1997

This is a preprint of a paper intended for publication in a journal or proceedings. Since changes may be made before publication, this preprint is made available with the understanding that it will not be cited or reproduced without the permission of the author. 


\section{DISCLAIMER}

This document was prepared as an account of work sponsored by an agency of the United States Government. Neither the United States Government nor the University of California nor any of their employees, makes any warranty, express or implied, or assumes any legal liability or responsibility for the accuracy, completeness, or usefulness of any information, apparatus, product, or process

disclosed, or represents that its use would not infringe privately owned rights. Reference herein to any specific commercial product, process, or service by trade name, trademark, manufacturer, or otherwise, does not necessarily constitute or imply its endorsement, recommendation, or favoring by the United States Government or the University of California. The views and opinions of authors expressed herein do not necessarily state or reflect those of the United States Government or the University of California, and shall not be used for advertising or product endorsement purposes. 


\title{
Color separation gratings for diverting the unconverted light away from the NIF target
}

\author{
S. N. Dixit, M. C. Rushford, I. M. Thomas, S. M. Herman, J. A. Britten, \\ B. W. Shore and M. D. Perry \\ Lawrence Livermore National Laboratory \\ Livermore, California 94550 \\ Tel: (510) 4237321 FAX (510) 422-5537 E-mail: dixit1@1lnl.gov
}

\section{INTRODUCTION}

Most of the glass laser based inertial confinement fusion systems around the world today employ non-linear frequency conversion for converting the $1.053 \mu \mathrm{m}$ light at the fundamental frequency (referred to as $1 \omega$ light) to either its second harmonic (called $2 \omega$ ) at $527 \mathrm{~nm}$ or to its third harmonic (called $3 \omega$ ) at $351 \mathrm{~nm}$. Shorter wavelengths are preferred for laser fusion because of the improved coupling of the laser light to the fusion targets due to reduced fast electron production at shorter wavelengths. The frequency conversion process, however, is only about $60-70 \%$ efficient and the residual $30-40 \%$ of the energy remains at $1 \omega$ and $2 \omega$ frequencies.

Different strategies have been adapted for diverting the unconverted light away from the fusion target. On the Nova laser system at Lawrence Livermore National Laboratory and on the Phebus laser system at Limeil, the chromatic aberration in the focusing lens is used to cast a shadow of a $15-\mathrm{cm}$ diameter circular obscuration at the center of the near-field profile to prevent the $1 \omega$ and $2 \omega$ light from hitting the target located at the $3 \omega$ focus. Here, different focal lengths at the $1 \omega$ and the $2 \omega$ wavelengths lead to scaleddown quasi far-field profiles of the input beam at the $3 \omega$ focal plane. The shadow of the obscuration then removes the unconverted light from the target. At the Omega laser system at the University of Rochester, the unconverted light is filtered out using the differing reflectivities of a multilayer turning mirror. Current baseline design for the proposed National Ignition Facility (NIF) employs a wedged final focusing lens to divert the unconverted $1 \omega$ and $2 \omega$ light away from the laser entrance hole and the outer surface of the hohlraum. The increased thickness of the glass in the UV beam path leads to higher nonlinear B-integral and thereby increasing the possible degradation in the beam quality and focussability of the $3 \omega$ light. In addition, larger deflections of the $1 \omega$ and $2 \omega$ light (if needed) would demand even thicker wedge on the lens, thus exacerbating the B-integral problem even more.

Color separation gratings ${ }^{1-5}$ (CSGs) offer a versatile approach to reducing and possibly eliminating the unconverted light at the target region. A CSG consists of a threelevel lamellar grating designed so that nearly all of the $3 \omega$ light passes through undiffracted while the residual $1 \omega$ and $2 \omega$ energy is diverted into higher diffraction orders. The diffraction angle is determined solely by the grating period. Since a CSG profile is only a few wavelengths deep, the CSG can be fabricated on a thinner optic. The reduced amount of fused silica in the beam path lowers the B integral thereby reducing the non-linear 
effects and consequently increasing the safety margin in the operation of high power fusion lasers such as NIF.

\section{CSG THEORY AND DESIGN CONSIDERATIONS}

In this section we will briefly outline the theory of color separation gratings and discuss the sensitivity of the CSG efficiency to various fabrication parameters. A schematic diagram of a color separation grating is shown in Fig. 1. It consists of a three level stair-case like lamellar structure. Although CSGs consisting of more than three levels can be designed, a minimum of three levels is required to achieve the color separation functionality desired on NIF. The height of each step is chosen so that a relative phase delay of $2 \pi$ is introduced for the $3 \omega$ light. If $d$ denotes the period of the grating, and $\lambda$ the operating wavelength, then the angle of diffraction for the $\mathrm{m}^{\text {th }}$ order when light is incident normal to the CSG is given by the standard grating equation

$$
\sin \left(\theta_{\mathrm{m}}(\lambda)\right)=\mathrm{m} \frac{\lambda}{\mathrm{d}} .
$$

Within the approximations of the scalar diffraction theory, the diffraction efficiencies in various orders are given by the following equation

$$
\eta_{m}=\left|\sum_{j=1-3} e^{-i \phi \phi_{n}^{n}\left(\lambda \lambda_{0}\right)-1} \frac{\lambda_{0}}{\lambda} e^{i 2 \pi m \varepsilon_{j}} \delta_{j} \operatorname{sinc}\left(\pi m \delta_{j}\right)\right|^{2}
$$

where $\phi_{\mathrm{j}}$ denotes the phase delay introduced due to the step $\mathrm{j}$ at the design wavelength $\lambda_{0}$ $(351 \mathrm{~nm})$, and $\varepsilon_{\mathrm{j}}$ and $\delta_{\mathrm{j}}$ denote the locations of the center and width of each zone within one CSG period. $\varepsilon_{\mathrm{j}}$ and $\delta_{\mathrm{j}}$ are normalized to the grating period. For a regularly spaced grating, $\delta_{j}=1 / 3$ for all $j$ and $\varepsilon_{j}=1 / 6,1 / 2$ and $5 / 6$ for $j=1,2$, and 3 . The factors multiplying the phase $\phi_{\mathrm{j}}$ account for the material dispersion and the chromatic dispersion due to the CSG profile.

If $\phi_{\mathrm{j}}=0,2 \pi$, and $4 \pi$ for $\lambda_{0}=351 \mathrm{~nm}$, then the grating appears transparent to the $3 \omega$ light and all the $3 \omega$ light passes through undiffracted in the zeroth transmitted order. Interestingly enough, this three level lamellar profile also implies a zero theoretical zeroth order efficiency for the $1 \omega$ and $2 \omega$ light in the absence of any material dispersion. The variation of the refractive index with wavelength leads to a small zeroth order diffraction efficiency for $1 \omega$ and $2 \omega$. Table 1 below lists the theoretical diffraction efficiencies for a few orders around 0 and for $1 \omega, 2 \omega$, and $3 \omega$ wavelengths. These results were based on the scalar theory (equation 2) which is valid for grating periods much larger than wavelength. In this limit, the diffraction efficiencies are independent of the CSG period and are insensitive to the polarization of the light.

It is clear from this table that about $88 \%$ of the $1 \omega$ light is diffracted into $+1,-2$ and +4 orders with the remaining energy distributed into many diffraction orders. The three-fold 
'skipping' of the diffraction efficiency is a consequence of the three step structure in the CSG period. It is also interesting to note that, while the $2 \omega$ efficiencies are approximately the same as the $1 \omega$ efficiencies, the sign of the diffraction order is switched between $1 \omega$ and $2 \omega$. This symmetry reversal results from the apparent reversal of the 'blaze' between $1 \omega$ and $2 \omega$. It can easily be shown that the step structure within a CSG period appears reversed for $2 \omega$ wavelength as compared to that for the $1 \omega$ wavelength.

\begin{tabular}{|c|c|c|c|}
\hline Diff. order & $1 \omega$ efficiency & $2 \omega$ efficiency & $3 \omega$ efficiency \\
\hline-4 & 0.0002 & 0.0422 & 0 \\
\hline-3 & 0 & 0 & 0 \\
\hline-2 & 0.169 & 0.001 & 0 \\
\hline-1 & 0.003 & 0.675 & 0 \\
\hline 0 & 0.005 & 0.006 & 0 \\
\hline 1 & 0.678 & 0.005 & 0 \\
\hline 2 & 0.0007 & 0.169 & 0 \\
\hline 3 & 0 & 0 & 0 \\
\hline 4 & 0.042 & 0.0003 & \\
\hline
\end{tabular}

Table 1. CSG diffraction efficiencies in various orders (equation 2). Refractive index values for fused silica were used in these calculations.

These are $n=1.44976,1.46097$ and 1.47672 at $1 \omega, 2 \omega$ and $3 \omega$ respectively.

The three level CSG profile has to be fabricated using a two mask lithographic process with either a wet etch or a dry etch into fused silica. The fabrication errors consist of errors in the etch depth and errors due to mask misalignment. Although the theoretical calculations above predict zeroth order diffraction efficiency of unity for the $3 \omega$ light and nearly zero for the residual $1 \omega$ and $2 \omega$ light, fabrication errors in a practical application reduce the throughput at $3 \omega$ and increase the $1 \omega$ and $2 \omega$ zero order light. Fig. 2 displays the variation of the diffraction efficiency in the zeroth order with etch depth. From these results we see that the etch depth has to be controlled to within $\pm 20 \mathrm{~nm}$ to maintain desirable $3 \omega$ focusing $(>98 \%)$ and color separation $(<3 \%)$ characteristics.

Mask misalignment and problems associated with undercutting during the lithographic fabrication of a CSG lead to an erosion of the sharpness of the edge between the various steps in the CSG. Types of erosion consist of a rounding off of the edge of the CSG grooves, a lateral shift in the location of the edge, a misaligned overlay etc. Often more than one type of error occurs in a given fabrication process. The dominant effect of these edge erosion errors is a loss of the $3 \omega$ zeroth order efficiency. This loss can be simply estimated as the fractional area loss due to the edge degradation. Detailed calculations confirm this. The $3 \omega$ zeroth order efficiency can then be written as 


$$
\eta_{0} \approx\left|1-\frac{\left(\Delta_{1}+\Delta_{2}+\Delta_{3}\right)}{\mathrm{d}}\right| \approx 1-2 \frac{\left(\Delta_{1}+\Delta_{2}+\Delta_{3}\right)}{\mathrm{d}}
$$

where $\Delta_{1}, \Delta_{2}$ and $\Delta_{3}$ denote widths of the eroded edges. Thus good alignment and edge definition control are required for maintaining high $3 \omega$ throughput. For example, for a CSG with a $300 \mu \mathrm{m}$ period, a $1 \mu \mathrm{m}$ misalignment at each step leads to a $2 \%$ loss of efficiency due to edge erosion. It should be mentioned that the $1 \omega$ and $2 \omega$ zeroth order efficiencies are less sensitive to the effects of edge degradation and etch depth errors.

\section{FABRICATION AND OPTICAL PERFORMANCE OF A CSG}

In order to demonstrate the concept of unconverted light management using color separation gratings, we fabricated a $\sim 120 \mathrm{~mm}$ diameter CSG having a $345 \mu \mathrm{m}$ period in a fused silica substrate using a lithographic process and wet etching ${ }^{6}$. This fabrication method is schematically illustrated in Fig. 3. The CSG substrate is first coated with a thin layer of chrome and a layer of photoresist is deposited over it. This photoresist layer is then exposed to ultraviolet light from an arc lamp through a binary (in transmission) photomask. The exposed photoresist is developed away exposing areas on the fused silica substrate that are etched in a buffered hydrofluoric acid solution. The etch depth is controlled by the time duration of the etch. Following the etching to a desired depth, the remaining photoresist is washed off. At this step one set of the CSG grooves has been fabricated. This entire sequence of steps is repeated for a second time with a second photomask to etch the second set of steps in the CSG substrate. The second mask pattern is aligned carefully to the first etched pattern in the substrate by using a set of precisely located alignment fiducials.

The binary photomasks required for the CSG fabrication were patterned in a chrome layer coated over a fused silica substrate using the large aperture photoplotter developed at our laboratory. The available plotting area for the photoplotter is about $1 \mathrm{~m} \times 1 \mathrm{~m}$ square. The pixel positioning and the pixel size are accurate to about $0.5 \mu \mathrm{m}$ each over the entire plotting area. We believe that the edge definition through the sequence of the lithographic steps mentioned above erodes to about $1.5 \mu \mathrm{m}$. This leads to a $\sim 2.5 \%$ reduction in the $3 \omega$ zeroth order throughput. The etch depths in fused silica were controlled to 10-15 $\mathrm{nm}$ at each etch step. This adds an additional $0.5 \%$ reduction to the $3 \omega$ efficiency.

The optical performance of the CSG was evaluated by measuring the diffraction efficiencies in the various orders generated by the CSG. The entire CSG was illuminated by coherent light at the $1 \omega, 2 \omega$ or $3 \omega$ wavelengths from the output of an Antares Nd: YLF laser. The line outs through the observed far-field $1 \omega, 2 \omega$ and $3 \omega$ intensity patterns are shown in Figure 4 . The diversion of the unconverted $1 \omega$ and $2 \omega$ light away from the zeroth order in the presence of a CSG is very clear. This figure also verifies the effectiveness of a CSG in maintaining a high $3 \omega$ zeroth order throughput.

In order to quantify the CSG performance, we measured the amount of light in various diffracted orders and find that greater than $95 \%$ of the incident light is preserved 
in the $3 \omega$ zeroth order while less than $5 \%$ of unconverted $1 \omega$ and $2 \omega$ light is remaining in the zeroth order.

\section{SUMMARY}

In summary, we have demonstrated the concept of using a color separation grating for diverting the unconverted light away from the target in high power fusion systems like the proposed National Ignition Facility. We fabricated a $345 \mu \mathrm{m}$ period CSG in fused silica using lithographic processes and wet etching. The measured far-field indicates that greater than $95 \%$ of the incident light is preserved in the $3 \omega$ zeroth order while less than $5 \%$ of unconverted $1 \omega$ and $2 \omega$ light is remaining in the zeroth order. Finally, we would like to add that diffractive optics fabricated in fused silica by wet etching in hydrofluoric acid should have high damage threshold. Our experience suggests that the damage threshold of the etched substrate is at least as high as the unetched part.

\section{ACKNOWLEDGEMENT}

This work was performed under the auspices of the U. S. Department of Energy by the Lawrence Livermore National Laboratory under Contract No. W-7405-ENG-48.

\section{REFERENCES}

1. H. Dammann, “Color separation gratings," Appl. Opt. 17 2273-2279 (1978).

2. T. Bett and I. Barton, AWE Aldermaston, Reading UK, private communication.

3. M. B. Stern and G. J. Swanson, "Color separation echelon gratings," in 1996 Technical Digest Series, vol. 5, Optical Society of America, April 1996.

4. M. W. Farn, M. B. Stern, W. B. Veldkamp, and S. S. Medeiros, "Color separation by use of binary optics," Opt. Lett. 181214 (1993).

5. M. W. Farn, R. E. Knowlden, M. B. Stern and W. B. Veldkamp, "Color separation gratings," NASA Conf Publ. 3227409 (1993).

6. See S. N. Dixit, I. M. Thomas, M. R. Rushford, R. Merrill, M. D. Perry, H. T. Powell and K. A. Nugent, "Kinoform phase plates for tailoring focal plane intensity profiles," pp 152-159 in ICF Quarterly Report, Lawrence Livermore National Laboratory, Livermore, CA, UCRL-LR105820-94 (1994). 


\section{Color separation grating (CSG)}

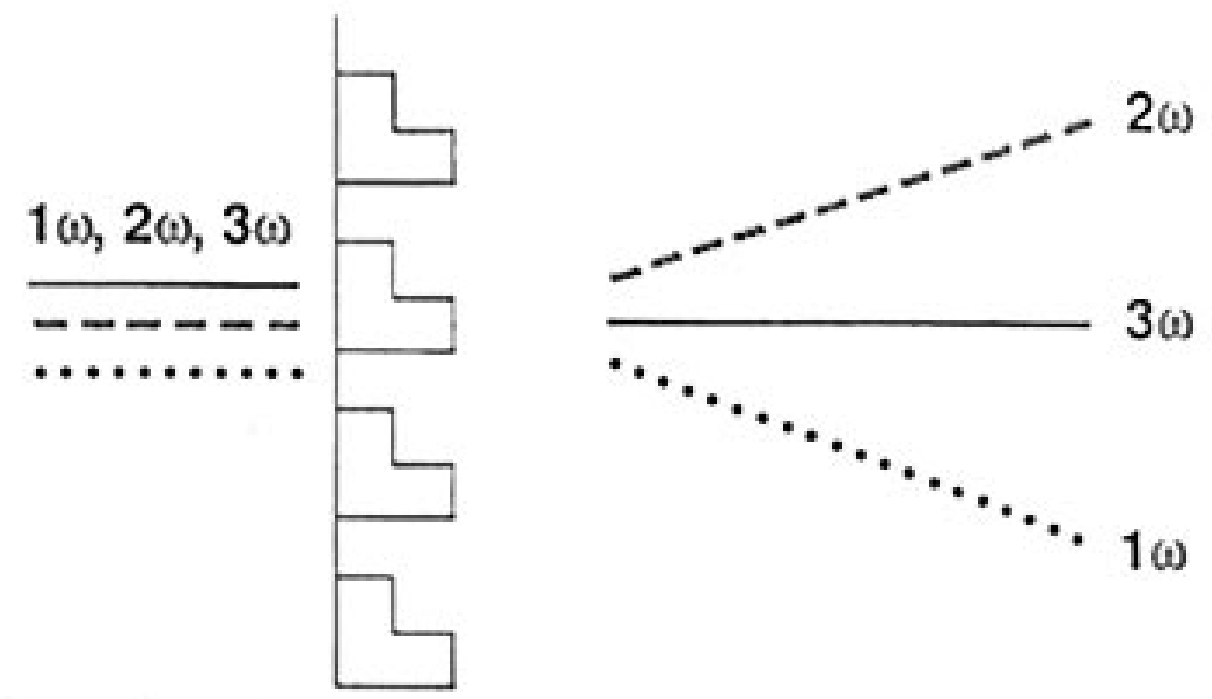

\section{Each step introduces a $2 \pi$ phase delay for $3 \omega$}

Fig. 1 Schematic illustration of the color separation grating concept.

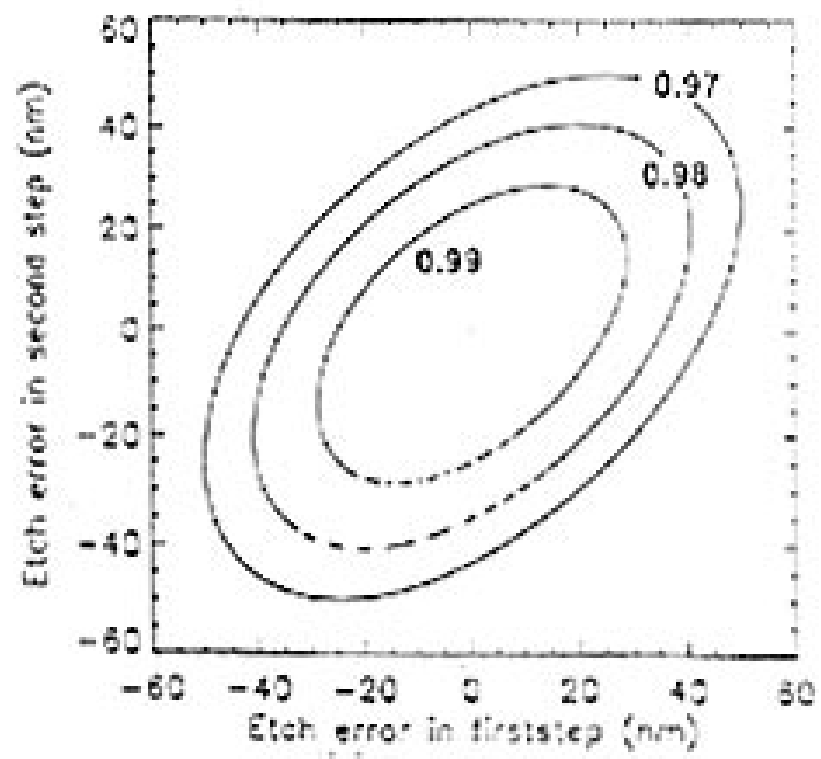

Fig. 2 A contour map of the zeroth order diffraction efficiency of a CSG as a function of the errors in the etch depths in the two steps. The values against each contour indicate the zeroth order diffraction efficiency. 


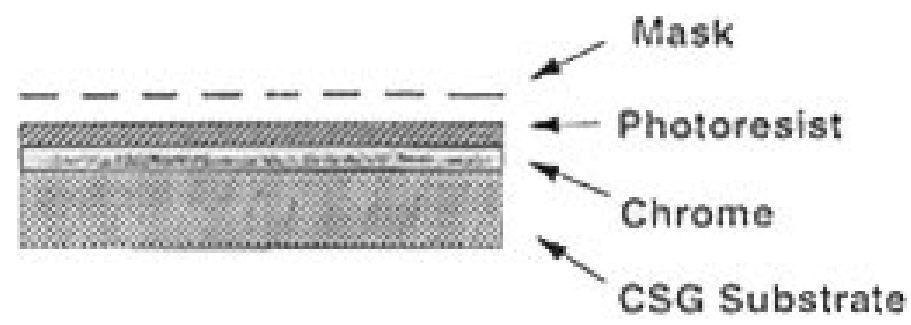

\section{Exposure}

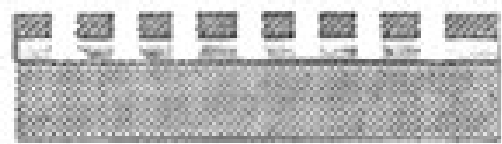

Develop

Chrome Etch

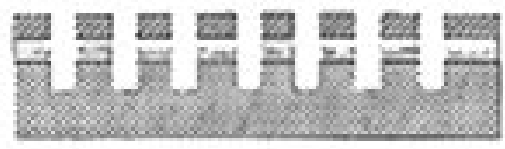

Wet Etch

Fig. 3 Illustration of the various steps in the fabrication of a CSG by lithographic methods. 

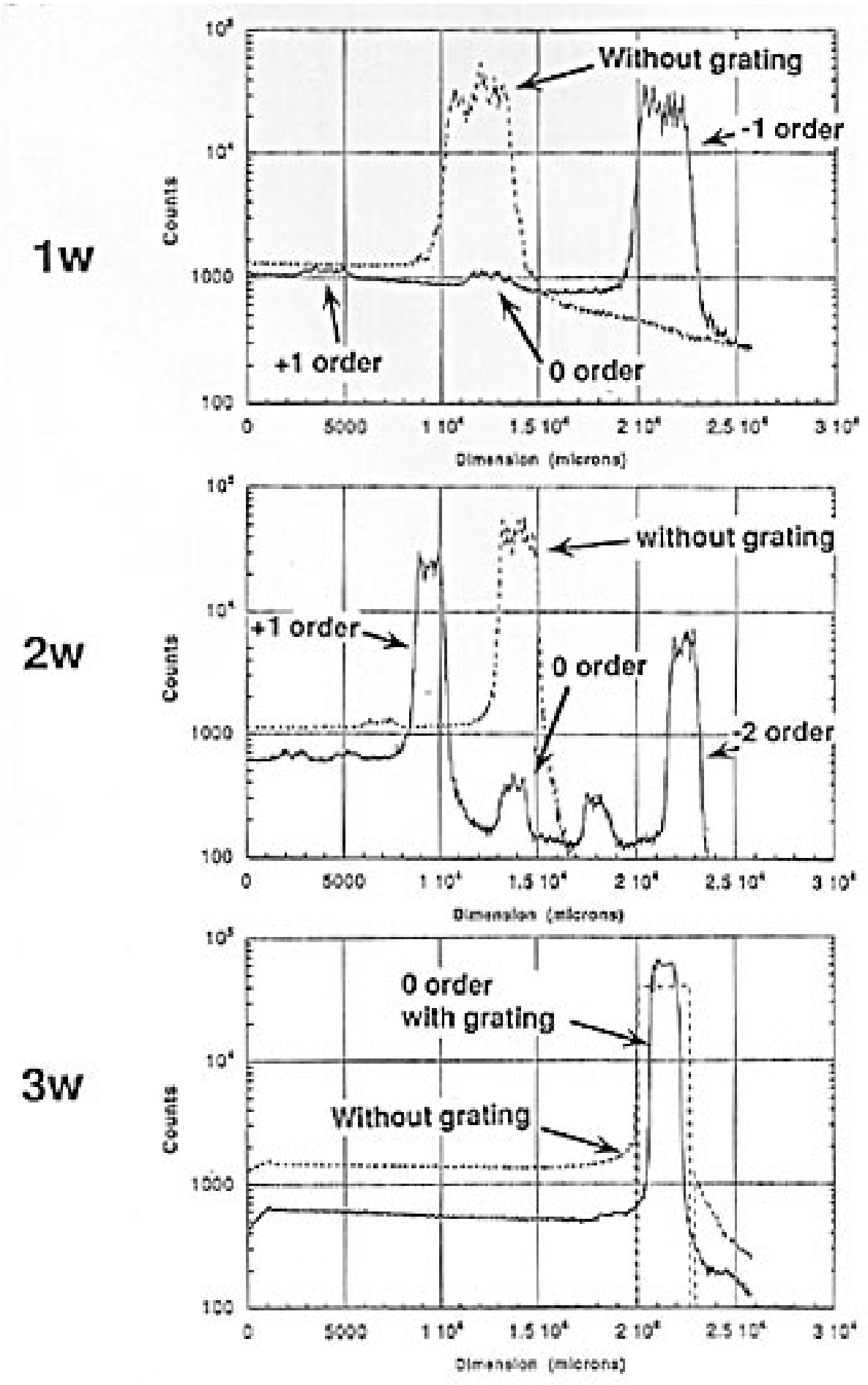

Fig. 4 Line outs through the experimentally observed $1 \omega, 2 \omega$ and $3 \omega$ diffracted orders produced by a CSG. The CSG period was $345 \mu \mathrm{m}$ and its aperture size was approximately 120 $\mathrm{mm}$. 


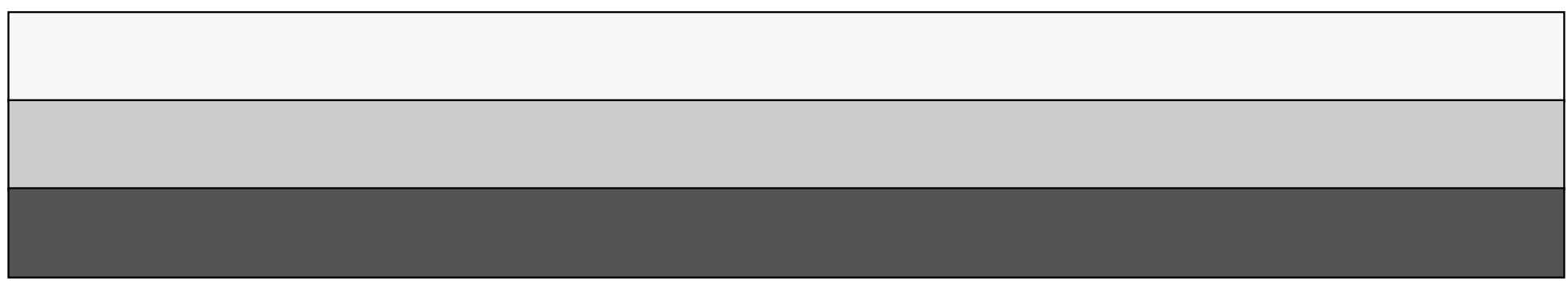

